\title{
Several new cyclic Jensen type inequalities and their applications
}

\author{
Nasir Mehmood', Saad Ihsan Butt ${ }^{1 *}$, Đilda Pečarić ${ }^{2}$ and Josip Pečarić ${ }^{3}$
}

\section{"Correspondence:}

saadihsanbutt@gmail.com

'Department of Mathematics, COMSATS University Islamabad, Lahore Campus, Pakistan Full list of author information is available at the end of the article

\begin{abstract}
We present some fundamental results and definitions regarding Jensen's inequality with the aim of obtaining new generalizations of cyclic refinements of Jensen's inequality from convex to higher order convex functions using Taylor's formula. We discuss the monotonicity of functionals for $n$-convex functions at a point. Applications of our work include new bounds for some important inequalities used in information theory.
\end{abstract}

MSC: Primary 26D10; secondary 26D20

Keywords: Jensen's inequality; Higher order convexity; Taylor's formula; New Green functions; Shannon entropy; Kullback-Leibler divergence; Zipf-Mandelbrot entropy

\section{Introduction}

The convexity of functions has been frequently used in various fields of pure and applied mathematics, for instance in function theory, mathematical analysis, functional analysis, probability theory, optimization theory, operational research, information theory. In short, convex functions entail a strong and elegant interaction between analysis and geometry. The simple generalization to a convex function extensively widens our scope for analysis.

Inequalities are frequently used in solving several problems of the applied sciences. Some recent work on the applications of mathematical inequalities can be found in [7, $12]$ and [24].

The Jensen inequality for convex functions plays a pivotal role in the theory of inequalities because of the fact that various other inequalities, for instance the Holder and Minkowski inequalities and the arithmetic mean-geometric mean inequality can be obtained as particular cases.

Some useful results on Jensen's inequality for strongly convex functions and generalized majorization inequalities can be found in [20] and [13].

For $\psi:[a, b] \rightarrow \mathbb{R}$ where $\psi^{(n-1)}$ is absolutely continuous, the renowned Taylor formula $\forall z \in[a, b]$ at the point $c \in[a, b]$ is

$$
\psi(z)=\sum_{w=0}^{n-1} \frac{\psi^{(w)}(c)}{w !}(z-c)^{w}+\frac{1}{(n-1) !} \int_{c}^{z} \psi^{(n)}(\xi)(z-\xi)^{n-1} d \xi
$$

(C) The Author(s) 2019. This article is distributed under the terms of the Creative Commons Attribution 4.0 International License (http://creativecommons.org/licenses/by/4.0/), which permits unrestricted use, distribution, and reproduction in any medium, provided you give appropriate credit to the original author(s) and the source, provide a link to the Creative Commons license, and indicate if changes were made. 
Taking $v=1, \ldots, 5$, the Green functions $G_{v}:[a, b] \times[a, b] \rightarrow \mathbb{R}$ are defined as

$$
\begin{aligned}
& G_{1}(z, \xi)= \begin{cases}\frac{(b-z)(a-\xi)}{b-a}, & a \leq \xi \leq z, \\
\frac{(b-\xi)(a-z)}{b-a}, & z \leq \xi \leq b,\end{cases} \\
& G_{2}(z, \xi)= \begin{cases}a-\xi, & a \leq \xi \leq z, \\
a-z, & z \leq \xi \leq b,\end{cases} \\
& G_{3}(z, \xi)= \begin{cases}z-b, & a \leq \xi \leq z, \\
\xi-b, & z \leq \xi \leq b,\end{cases} \\
& G_{4}(z, \xi)= \begin{cases}z-a, & a \leq \xi \leq z, \\
\xi-a, & z \leq \xi \leq b,\end{cases} \\
& G_{5}(z, \xi)= \begin{cases}b-\xi, & a \leq \xi \leq z, \\
b-z, & z \leq \xi \leq b .\end{cases}
\end{aligned}
$$

Each of these functions is convex and continuous with respect to the two variables $z$ and $\xi$.

Lemma 1 ([19]) Suppose $\psi \in C^{2}[a, b]$, then the following identities are valid:

$$
\begin{aligned}
& \psi(z)=\frac{a-z}{b-a} \psi(a)+\frac{z-a}{b-a} \psi(b)+\int_{a}^{b} G_{1}(z, \xi) \psi^{\prime \prime}(\xi) d \xi, \\
& \psi(z)=\psi(a)+(z-a) \psi^{\prime}(b)+\int_{a}^{b} G_{2}(z, \xi) \psi^{\prime \prime}(\xi) d \xi, \\
& \psi(z)=\psi(b)+(b-z) \psi^{\prime}(a)+\int_{a}^{b} G_{3}(z, \xi) \psi^{\prime \prime}(\xi) d \xi, \\
& \psi(z)=\psi(b)-(b-a) \psi^{\prime}(b)+(z-a) \psi^{\prime}(a)+\int_{a}^{b} G_{4}(z, \xi) \psi^{\prime \prime}(\xi) d \xi, \\
& \psi(z)=\psi(a)+(b-a) \psi^{\prime}(a)-(b-z) \psi^{\prime}(b)+\int_{a}^{b} G_{5}(z, \xi) \psi^{\prime \prime}(\xi) d \xi .
\end{aligned}
$$

Proof Consider the integral

$$
\int_{a}^{b} G_{\nu}(z, r) \psi^{\prime \prime}(r) d r=\int_{a}^{z} G_{\nu}(z, r) \psi^{\prime \prime}(r) d r+\int_{z}^{b} G_{v}(z, r) \psi^{\prime \prime}(r) d r
$$

For fixed $v=1, \ldots, 5$, after integrating for a particular value of the Green functions, (7)(11) are obtained.

In order to state some recent investigations of Jensen's inequality, we shall use the following assumptions:

$\left(\mathrm{H}_{1}\right)$ Suppose $2 \leq k \leq m$ are integers and probability distributions $\mathbf{p}, \boldsymbol{\lambda} \in \mathbb{R}_{+}^{m}$ so that $\sum_{i} p_{i}=1$ and $\sum_{k} \lambda_{k}=1$.

$\left(\mathrm{H}_{2}\right)$ Suppose $(Z, \mathcal{B}, \mu)$ represents probability space. 
Suppose for a fixed integer $l \geq 2$ and for $m=1, \ldots, l, \mathcal{B}^{l}$ denotes the $\sigma$-algebra in $Z^{l}$ which is generated by the projection mappings $p_{m}: Z^{l} \rightarrow Z$ given as

$$
p r_{m}\left(z_{1}, \ldots, z_{l}\right):=z_{m}
$$

where $\mu^{l}$ represents a product measure on $\mathcal{B}^{l}$. This measure is uniquely ( $\mu$ is $\sigma$ finite) specified by

$$
\mu^{l}\left(B_{1} \times \cdots \times B_{l}\right):=\mu\left(B_{1}\right) \cdots \mu\left(B_{l}\right), \quad B_{m} \in \mathcal{B}, m=1, \ldots, l
$$

$\left(\mathrm{H}_{3}\right) g$ is $\mu$-integrable on $Z$ with values in $I \subset \mathbb{R}$.

$\left(\mathrm{H}_{4}\right)$ Suppose $\psi$ is convex on $I$ with $\psi \circ g$ being $\mu$-integrable on $Z$.

Considering the assumptions $\left(\mathrm{H}_{1}\right)-\left(\mathrm{H}_{4}\right)$, we now define the following.

Theorem 1 ([8]) Assume $\left(\mathrm{H}_{1}\right)$ with $\psi: C \rightarrow \mathbb{R}$ being a convex function where $C$ is a convex subset of real vector space $Z$. If $z_{1}, \ldots, z_{m} \in C$, then

$$
\begin{aligned}
\psi\left(\sum_{i=1}^{m} p_{i} z_{i}\right) & \leq C_{\mathrm{dis}}=C_{\mathrm{dis}}(\psi, z, \mathbf{p}, \lambda) \\
& :=\sum_{i=1}^{m}\left(\sum_{j=0}^{k-1} \lambda_{j+1} p_{i+j}\right) \psi\left(\frac{\sum_{j=0}^{k-1} \lambda_{j+1} p_{i+j} z_{i+j}}{\sum_{j=0}^{k-1} \lambda_{j+1} p_{i+j}}\right) \leq \sum_{i=1}^{m} p_{i} \psi\left(z_{i}\right),
\end{aligned}
$$

while $i+j$ means $i+j(\bmod m)$.

Theorem 2 ([8]) Suppose $\left(\mathrm{H}_{1}\right)-\left(\mathrm{H}_{4}\right)$ hold. Then

$$
\psi\left(\int_{Z} g d \mu\right) \leq C_{\mathrm{par}}(t) \leq C_{\mathrm{int}} \leq \int_{Z} \psi \circ g d \mu, \quad t \in[0,1],
$$

where

$$
\begin{aligned}
C_{\mathrm{int}} & =C_{\mathrm{int}}(\psi, g, \mu, \mathbf{p}, \lambda) \\
& :=\sum_{i=1}^{m}\left(\sum_{j=0}^{k-1} \lambda_{j+1} p_{i+j}\right) \int_{Z^{m}} \psi\left(\frac{\sum_{j=0}^{k-1} \lambda_{j+1} p_{i+j} g\left(z_{i+j}\right)}{\sum_{j=0}^{k-1} \lambda_{j+1} p_{i+j}}\right) d \mu^{m}\left(z_{1}, \ldots, z_{m}\right),
\end{aligned}
$$

and for $t \in[0,1]$

$$
\begin{aligned}
C_{\mathrm{par}}(t)= & C_{\mathrm{par}}(t, \psi, g, \mu, \mathbf{p}, \lambda) \\
:= & \sum_{i=1}^{m}\left(\sum_{j=0}^{k-1} \lambda_{j+1} p_{i+j}\right) \\
& \cdot \int_{Z^{m}} \psi\left(t \frac{\sum_{j=0}^{k-1} \lambda_{j+1} p_{i+j} g\left(z_{i+j}\right)}{\sum_{j=0}^{k-1} \lambda_{j+1} p_{i+j}}+(1-t) \int_{Z} g d \mu\right) d \mu^{m}\left(z_{1}, \ldots, z_{m}\right),
\end{aligned}
$$

while $i+j$ means $i+j(\bmod m)$. 
For the purpose of achieving our targets, we construct non-negative linear functionals from the above theorem.

Remark 1 Under the assumptions of Theorem 1 and Theorem 2 for $\psi$ to be a convex function, we have

$$
\begin{aligned}
& J_{1}(\psi)=J_{1}(\mathbf{z}, \mathbf{p}, \lambda ; \psi):=\sum_{i=1}^{m} p_{i} \psi\left(z_{i}\right)-C_{\mathrm{dis}}(\psi, \mathbf{z}, \mathbf{p}, \lambda) \geq 0, \\
& J_{2}(\psi)=J_{1}(\mathbf{z}, \mathbf{p}, \lambda ; \psi):=C_{\mathrm{dis}}(\psi, \mathbf{z}, \mathbf{p}, \lambda)-\psi\left(\sum_{i=1}^{m} p_{i} z_{i}\right) \geq 0, \\
& J_{3}(\psi)=J_{3}(\psi, g, \mu, \mathbf{p}, \lambda):=\int_{X} \psi \circ g d \mu-C_{\text {int }}(\psi, g, \mu, \mathbf{p}, \lambda) \geq 0, \\
& J_{4}(\psi)=J_{4}(t, \psi, g, \mu, \mathbf{p}, \lambda):=\int_{X} \psi \circ g d \mu-C_{\mathrm{par}}(t, \psi, g, \mu, \mathbf{p}, \lambda) \geq 0 ; \quad t \in[0,1], \\
& J_{5}(\psi)=J_{5}(t, \psi, g, \mu, \mathbf{p}, \lambda):=C_{\mathrm{int}}(\psi, g, \mu, \mathbf{p}, \lambda)-C_{\mathrm{par}}(t, \psi, g, \mu, \mathbf{p}, \lambda) \geq 0 ; \quad t \in[0,1], \\
& J_{6}(\psi)=J_{6}(t, \psi, g, \mu, \mathbf{p}, \lambda):=C_{\mathrm{par}}(t, \psi, g, \mu, \mathbf{p}, \lambda)-\psi\left(\int_{X} g d \mu\right) \geq 0 ; \quad t \in[0,1] .
\end{aligned}
$$

\section{Main results}

To start for real weights, we need the following assumptions:

$\left(\mathrm{A}_{1}\right)$ For $J_{u}(\cdot)(u=1,2)$, suppose $\frac{\sum_{j=0}^{k-1} \lambda_{j+1} p_{i+j} z_{i+j}}{\sum_{j=0}^{k-1} \lambda_{j+1} p_{i+j}} \in[a, b]$ for $i=1, \ldots, m$.

$\left(\mathrm{A}_{2}\right)$ For the linear functionals $J_{u}(\cdot)(u=3, \ldots, 6)$, suppose $\left(\mathrm{H}_{2}\right)-\left(\mathrm{H}_{4}\right)$ are satisfied and

$$
\frac{\sum_{j=0}^{k-1} \lambda_{j+1} p_{i+j} g\left(z_{i+j}\right)}{\sum_{j=0}^{k-1} \lambda_{j+1} p_{i+j}} \in[a, b] \text { for } i=1, \ldots, m
$$

We take cyclic refinements of Jensen's inequality in discrete and continuous versions and form the following identities with real weights by using Taylor's formula.

Theorem 3 Suppose $m, k \in \mathbb{N}, p_{1}, \ldots, p_{m}$ and $\lambda_{1}, \ldots, \lambda_{k}$ are real tuples for $2 \leq k \leq m$, such that $\sum_{j=0}^{k-1} \lambda_{j+1} p_{i+j} \neq 0$ for $i=1, \ldots, m$ with $\sum_{i=1}^{m} p_{i}=1$ and $\sum_{j=1}^{k} \lambda_{j}=1$. Alsolet $z \in[a, b] \subset \mathbb{R}$ and $\mathbf{z} \in[a, b]^{m}$. Consider the function $\psi:[a, b] \rightarrow \mathbb{R}$ such that $\psi^{(n-1)}$ is absolutely continuous and $G_{v}(v=1, \ldots, 5)$ are same as given in (2)-(6), respectively. Then for $(u=1, \ldots, 6)$ along with the assumptions $\left(\mathrm{A}_{1}\right)$ and $\left(\mathrm{A}_{2}\right)$, we have the following generalized identities:

(a)

$$
J_{u}(\psi)=\sum_{w=1}^{n-1} \frac{\psi^{(w)}(a)}{w !} J_{u}\left((z-a)^{w}\right)+\frac{1}{(n-1) !} \int_{a}^{b} \psi^{(n)}(\xi) J_{u}\left((z-\xi)_{+}^{n-1}\right) d \xi
$$

(b)

$$
J_{u}(\psi)=\sum_{w=1}^{n-1} \frac{(-1)^{w} \psi^{(w)}(b)}{w !} J_{u}\left((b-z)^{w}\right)-\frac{(-1)^{n-1}}{(n-1) !} \int_{a}^{b} \psi^{(n)}(\xi) J_{u}\left((\xi-z)_{+}^{n-1}\right) d \xi
$$


(c)

$$
\begin{aligned}
J_{u}(\psi)= & \left(\frac{\psi(b)-\psi(a)}{b-a}\right) J_{u}(z)+\int_{a}^{b} J_{u}\left(G_{1}(z, r)\right)\left(\sum_{w=2}^{n-1} \frac{\psi^{(w)}(a)(r-a)^{w-2}}{(w-2) !}\right) d r \\
& +\frac{1}{(n-3) !} \int_{a}^{b} \psi^{(n)}(\xi)\left(\int_{\xi}^{b} J_{u}\left(G_{1}(z, r)\right)(r-\xi)^{n-3} d r\right) d \xi, \\
J_{u}(\psi)= & \psi^{\prime}(b) J_{u}(z)+\int_{a}^{b} J_{u}\left(G_{2}(z, r)\right)\left(\sum_{w=2}^{n-1} \frac{\psi^{(w)}(a)(r-a)^{w-2}}{(w-2) !}\right) d r \\
& +\frac{1}{(n-3) !} \int_{a}^{b} \psi^{(n)}(\xi)\left(\int_{\xi}^{b} J_{u}\left(G_{2}(z, r)\right)(r-\xi)^{n-3} d r\right) d \xi, \\
J_{u}(\psi)= & -\psi^{\prime}(a) J_{u}(z)+\int_{a}^{b} J_{u}\left(G_{3}(z, r)\right)\left(\sum_{w=2}^{n-1} \frac{\psi^{(w)}(a)(r-a)^{w-2}}{(w-2) !}\right) d r \\
& +\frac{1}{(n-3) !} \int_{a}^{b} \psi^{(n)}(\xi)\left(\int_{\xi}^{b} J_{u}\left(G_{3}(z, r)\right)(r-\xi)^{n-3} d r\right) d \xi, \\
J_{u}(\psi)= & \psi^{\prime}(a) J_{u}(z)+\int_{a}^{b} J_{u}\left(G_{4}(z, r)\right)\left(\sum_{w=2}^{n-1} \frac{\psi^{(w)}(a)(r-a)^{w-2}}{(w-2) !}\right) d r \\
& +\frac{1}{(n-3) !} \int_{a}^{b} \psi^{(n)}(\xi)\left(\int_{\xi}^{b} J_{u}\left(G_{4}(z, r)\right)(r-\xi)^{n-3} d r\right) d \xi, \\
& +\frac{1}{(n-3) !} \int_{a}^{b} \psi^{(n)}(\xi)\left(\int_{\xi}^{b} J_{u}\left(G_{5}(z, r)\right)(r-\xi)^{n-3} d r\right) d \xi, \\
J_{u}(\psi)= & \psi^{\prime}(b) J_{u}(z)+\int_{a}^{b} J_{u}\left(G_{5}(z, r)\right)\left(\sum_{w=2}^{n-1} \frac{\psi^{(w)}(a)(r-a)^{w-2}}{(w-2) !}\right) d r
\end{aligned}
$$

(d)

$$
\begin{aligned}
J_{u}(\psi)= & \left(\frac{\psi(b)-\psi(a)}{b-a}\right) J_{u}(z)+\int_{a}^{b} J_{u}\left(G_{1}(z, r)\right)\left(\sum_{w=2}^{n-1} \frac{\psi^{(w)}(a)(r-b)^{w-2}}{(w-2) !}\right) d r \\
& -\frac{1}{(n-3) !} \int_{a}^{b} \psi^{(n)}(\xi)\left(\int_{a}^{\xi} J_{u}\left(G_{1}(z, r)\right)(r-\xi)^{n-3} d r\right) d \xi, \\
J_{u}(\psi)= & \psi^{\prime}(b) J_{u}(z)+\int_{a}^{b} J_{u}\left(G_{2}(z, r)\right)\left(\sum_{w=2}^{n-1} \frac{\psi^{(w)}(a)(r-b)^{w-2}}{(w-2) !}\right) d r \\
& -\frac{1}{(n-3) !} \int_{a}^{b} \psi^{(n)}(\xi)\left(\int_{a}^{\xi} J_{u}\left(G_{2}(z, r)\right)(r-\xi)^{n-3} d r\right) d \xi, \\
J_{u}(\psi)= & -\psi^{\prime}(a) J_{u}(z)+\int_{a}^{b} J_{u}\left(G_{3}(z, r)\right)\left(\sum_{w=2}^{n-1} \frac{\psi^{(w)}(a)(r-b)^{w-2}}{(w-2) !}\right) d r \\
& -\frac{1}{(n-3) !} \int_{a}^{b} \psi^{(n)}(\xi)\left(\int_{a}^{\xi} J_{u}\left(G_{3}(z, r)\right)(r-\xi)^{n-3} d r\right) d \xi, \\
J_{u}(\psi)= & \psi^{\prime}(a) J_{u}(z)+\int_{a}^{b} J_{u}\left(G_{4}(z, r)\right)\left(\sum_{w=2}^{n-1} \frac{\psi^{(w)}(a)(r-b)^{w-2}}{(w-2) !}\right) d r \\
& -\frac{1}{(n-3) !} \int_{a}^{b} \psi^{(n)}(\xi)\left(\int_{a}^{\xi} J_{u}\left(G_{4}(z, r)\right)(r-\xi)^{n-3} d r\right) d \xi,
\end{aligned}
$$




$$
\begin{aligned}
J_{u}(\psi)= & \psi^{\prime}(b) J_{u}(z)+\int_{a}^{b} J_{u}\left(G_{5}(z, r)\right)\left(\sum_{w=2}^{n-1} \frac{\psi^{(w)}(a)(r-b)^{w-2}}{(w-2) !}\right) d r \\
& -\frac{1}{(n-3) !} \int_{a}^{b} \psi^{(n)}(\xi)\left(\int_{a}^{\xi} J_{u}\left(G_{5}(z, r)\right)(r-\xi)^{n-3} d r\right) d \xi,
\end{aligned}
$$

where $(z-\xi)_{+}=\max (z-\xi, 0)$.

Proof Fix $u=1, \ldots, 6$.

(a) Applying Taylor's formula (1) at point $a$, we get

$$
\psi(z)=\sum_{w=0}^{n-1} \frac{\psi^{(w)}(a)}{w !}(z-a)^{w}+\frac{1}{(n-1) !} \int_{a}^{b} \psi^{(n)}(\xi)(z-\xi)_{+}^{n-1} d \xi
$$

Since each $J_{u}(\cdot)$ is a linear functional, applying it to the Taylor expression (27) and using the constancy property of the $J_{u}(\cdot)$, we get (15).

(b) Applying Taylor's formula (1) at point $b$, we get

$$
\psi(z)=\sum_{w=0}^{n-1} \frac{(-1)^{w} \psi^{(w)}(b)}{w !}(z-b)^{w}-\frac{(-1)^{n-1}}{(n-1) !} \int_{a}^{b} \psi^{(n)}(\xi)(\xi-z)_{+}^{n-1} d \xi
$$

and following similar steps we get (16).

(c) For fixed $v=1$, applying (7) to $J_{u}(\cdot)$ and using the linearity of $J_{u}(\cdot)$, we have

$$
\begin{aligned}
J_{u}(\psi)= & \psi(a) J_{u}\left(\frac{b-z}{b-a}\right)+\psi(b) J_{u}\left(\frac{z-a}{b-a}\right)+\int_{a}^{b} J_{u}\left(G_{1}(z, r)\right) \psi^{\prime \prime}(r) d r \\
= & \psi(a) \frac{J_{u}(b-z)}{b-a}+\psi(b) \frac{J_{u}(z-a)}{b-a}+\int_{a}^{b} J_{u}\left(G_{1}(z, r)\right) \psi^{\prime \prime}(r) d r \\
= & \frac{1}{b-a}\left(\psi(a) J_{u}(b)-\psi(a) J_{u}(z)+\psi(b) J_{u}(z)-\psi(b) J_{u}(a)\right) \\
& +\int_{a}^{b} J_{u}\left(G_{1}(z, r)\right) \psi^{\prime \prime}(r) d r \\
= & \frac{1}{b-a}\left(\psi(b) J_{u}(z)-\psi(a) J_{u}(z)\right)+\int_{a}^{b} J_{u}\left(G_{1}(z, r)\right) \psi^{\prime \prime}(r) d r .
\end{aligned}
$$

Differentiating (1) twice and putting $c=a$ and replacing $n$ by $(n-2)$ or utilizing $(1)$ on the function $\psi^{\prime \prime}$ at the point $a$, we get

$$
\psi^{\prime \prime}(r)=\sum_{w=2}^{n-1} \frac{\psi^{(w)}(a)}{(w-2) !}(r-a)^{w-2}+\frac{1}{(n-3) !} \int_{a}^{r} \psi^{(n)}(\xi)(r-\xi)^{n-3} d \xi
$$

Now, using (30) in (29), we get

$$
\begin{aligned}
J_{u}(\psi)= & \left(\frac{\psi(b)-\psi(a)}{b-a}\right) J_{u}(z)+\int_{a}^{b} J_{u}\left(G_{1}(z, r)\right)\left(\sum_{w=2}^{n-1} \frac{\psi^{(w)}(a)(r-a)^{w-2}}{(w-2) !}\right) d r \\
& +\frac{1}{(n-3) !} \int_{a}^{b} J_{u}\left(G_{1}(z, r)\right)\left(\int_{a}^{r} \psi^{(n)}(\xi)(r-\xi)^{n-3} d \xi\right) d r .
\end{aligned}
$$


Now applying Fubini's theorem to the last term gives (17), respectively, for $v=1$ and $u=$ $1, \ldots, 6$. The results for $v=2,3,4,5$ can be obtained in the same way.

(d) Differentiating (1) twice and now taking $c=b$, we get

$$
\psi^{\prime \prime}(r)=\sum_{w=2}^{n-1} \frac{\psi^{(w)}(b)}{(w-2) !}(r-b)^{w-2}-\frac{1}{(n-3) !} \int_{r}^{b} \psi^{(n)}(\xi)(r-\xi)^{n-3} d \xi
$$

Analogously, putting (31) in (29) and using Fubini's theorem gives (22), respectively, for $v=1$ and $u=1, \ldots, 6$. The results for $v=2,3,4,5$ can be obtained in the same way.

The following theorem gives a key criterion to test the $n$-convexity of a function $\psi$ (see $[23$, p. 16]).

Theorem 4 If $\psi^{(n)}$ exists, then $\psi$ is $n$-convex if and only if $\psi^{(n)} \geq 0$.

We now use Theorem 4 to give generalizations of cyclic Jensen type linear functionals for real weights.

Corollary 1 Under the assumptions of Theorem 3, if $\psi$ is n-convex, we obtain the following results:

(a) If, for all $u=1, \ldots, 6$,

$$
J_{u}\left((z-\xi)_{+}^{n-1}\right) \geq 0, \quad \xi \in[a, b]
$$

holds, then we have

$$
J_{u}(\psi) \geq \sum_{w=1}^{n-1} \frac{\psi^{(w)}(a)}{w !} J_{u}\left((z-a)^{w}\right)
$$

for $u=1, \ldots, 6$

(b) If, for all $u=1, \ldots, 6$,

$$
(-1)^{n-1} J_{u}\left((\xi-z)_{+}^{n-1}\right) \leq 0, \quad \xi \in[a, b],
$$

holds, then we have

$$
J_{u}(\psi) \geq \sum_{w=1}^{n-1} \frac{(-1)^{w} \psi^{(w)}(b)}{w !} J_{u}\left((b-z)^{w}\right)
$$

for $u=1, \ldots, 6$.

(c) If, for all $u=1, \ldots, 6$ and $v=1, \ldots, 5$,

$$
\int_{\xi}^{b} J_{u}\left(G_{v}(z, r)\right)(r-\xi)^{n-3} d r \geq 0, \quad \xi \in[a, b]
$$


holds, then we have

$$
\begin{aligned}
J_{u}(\psi) \geq & \left(\frac{\psi(b)-\psi(a)}{b-a}\right) J_{u}(z) \\
& +\int_{a}^{b} J_{u}\left(G_{1}(z, r)\right)\left(\sum_{w=2}^{n-1} \frac{\psi^{(w)}(a)(r-a)^{w-2}}{(w-2) !}\right) d r \\
J_{u}(\psi) \geq & \psi^{\prime}(b) J_{u}(z)+\int_{a}^{b} J_{u}\left(G_{2}(z, r)\right)\left(\sum_{w=2}^{n-1} \frac{\psi^{(w)}(a)(r-a)^{w-2}}{(w-2) !}\right) d r \\
J_{u}(\psi) \geq & -\psi^{\prime}(a) J_{u}(z)+\int_{a}^{b} J_{u}\left(G_{3}(z, r)\right)\left(\sum_{w=2}^{n-1} \frac{\psi^{(w)}(a)(r-a)^{w-2}}{(w-2) !}\right) d r \\
J_{u}(\psi) \geq & \psi^{\prime}(a) J_{u}(z)+\int_{a}^{b} J_{u}\left(G_{4}(z, r)\right)\left(\sum_{w=2}^{n-1} \frac{\psi^{(w)}(a)(r-a)^{w-2}}{(w-2) !}\right) d r \\
J_{u}(\psi) \geq & \psi^{\prime}(b) J_{u}(z)+\int_{a}^{b} J_{u}\left(G_{5}(z, r)\right)\left(\sum_{w=2}^{n-1} \frac{\psi^{(w)}(a)(r-a)^{w-2}}{(w-2) !}\right) d r .
\end{aligned}
$$

(d) If, for all $u=1, \ldots, 6$ and $v=1, \ldots, 5$,

$$
\int_{a}^{\xi} J_{u}\left(G_{\nu}(z, r)\right)(r-\xi)^{n-3} d r \leq 0, \quad \xi \in[a, b]
$$

holds, then we have

$$
\begin{aligned}
& J_{u}(\psi) \geq\left(\frac{\psi(b)-\psi(a)}{b-a}\right) J_{u}(z)+\int_{a}^{b} J_{u}\left(G_{1}(z, r)\right)\left(\sum_{w=2}^{n-1} \frac{\psi^{(w)}(b)(r-b)^{w-2}}{(w-2) !}\right) d r, \\
& J_{u}(\psi) \geq \psi^{\prime}(b) J_{u}(z)+\int_{a}^{b} J_{u}\left(G_{2}(z, r)\right)\left(\sum_{w=2}^{n-1} \frac{\psi^{(w)}(b)(r-b)^{w-2}}{(w-2) !}\right) d r \\
& J_{u}(\psi) \geq-\psi^{\prime}(a) J_{u}(z)+\int_{a}^{b} J_{u}\left(G_{3}(z, r)\right)\left(\sum_{w=2}^{n-1} \frac{\psi^{(w)}(b)(r-b)^{w-2}}{(w-2) !}\right) d r, \\
& J_{u}(\psi) \geq \psi^{\prime}(a) J_{u}(z)+\int_{a}^{b} J_{u}\left(G_{4}(z, r)\right)\left(\sum_{w=2}^{n-1} \frac{\psi^{(w)}(b)(r-b)^{w-2}}{(w-2) !}\right) d r \\
& J_{u}(\psi) \geq \psi^{\prime}(b) J_{u}(z)+\int_{a}^{b} J_{u}\left(G_{5}(z, r)\right)\left(\sum_{w=2}^{n-1} \frac{\psi^{(w)}(b)(r-b)^{w-2}}{(w-2) !}\right) d r .
\end{aligned}
$$

Proof We begin with the proof of (a) and its assumed conditions. Fix $u=1, \ldots, 6$.

By our assumption $\psi^{(n-1)}$ is absolutely continuous on $[a, b]$, as a result $\psi^{(n)}$ exists almost everywhere. Moreover, $\psi$ is supposed to be $n$-convex, so by Theorem $4, \psi^{(n)}(z) \geq 0$ almost everywhere on $[a, b]$. Therefore by applying Theorem 3 , we get (33).

Similarly, the rest of the inequalities can be proved.

We now give the final results of the present section. 
Theorem 5 Under the assumptions of Theorem 3 if $p_{1}, \ldots, p_{m}$ and $\lambda_{1}, \ldots, \lambda_{k}$ are nonnegative tuples such that $\sum_{i=1}^{m} p_{i}=1$ and $\sum_{j=1}^{k} \lambda_{j}=1$, then for $\psi$ being $n$-convex, we have:

(a) Inequality (33) is valid when $n \geq 3$. Besides, for the function

$$
F_{1}(z):=\sum_{w=1}^{n-1} \frac{\psi^{(w)}(a)}{w !}(z-a)^{w}
$$

to be convex, the right side of (33) is non-negative, meaning

$$
J_{u}(\psi) \geq 0, \quad u=1, \ldots, 6
$$

(b) For n even, (35) holds. Furthermore, for the function

$$
F_{2}(z):=\sum_{w=1}^{n-1} \frac{(-1)^{w} \psi^{(w)}(b)}{w !}(b-z)^{w}
$$

to be convex, the right hand side of (35) is non-negative, particularly (49) holds.

(c) Inequalities (37)-(41) hold for all $n \geq 3$. Moreover, let (37)-(41) be valid and

$$
\sum_{w=2}^{n-1} \frac{\psi^{(w)}(a)(r-a)^{w-2}}{(w-2) !} \geq 0,
$$

then we get (49) for every $u=1, \ldots, 6$ and $v=1, \ldots, 5$.

(d) If $n$ is even, then (43)-(47) hold. Moreover, let (43)-(47) be valid and

$$
\sum_{w=2}^{n-1} \frac{\psi^{(w)}(b)(r-a)^{w-2}}{(w-2) !} \geq 0
$$

then we get (49) for $u=1, \ldots, 6$ and for $v=1, \ldots, 5$.

Proof (a) For fixed $u=1, \ldots, 6$.

For ( $n \geq 3), x \mapsto\left((x-t)_{+}\right)^{n-1}$ is a convex function, so (32) holds by virtue of Remark 1 on account of the given weights being positive. Hence (33) is established by taking into account Corollary 1(a). Moreover, we can write the R.H.S. of (33) in the form $J_{u}\left(F_{1}\right)$ for $(u=$ $1, \ldots, 6)$, after reorganizing this side. By using Remark 1 we can ensure the non-negativity of the R.H.S. of (33), especially (49) is established.

(b) Similar to the proof of (a).

(c) Fix $u=1, \ldots, 6$.

Considering the positive weights and $v=1, \ldots, 5, G_{v}(z, r)$ is convex. Hence using Remark $1, J_{u}\left(G_{v}(z, r)\right) \geq 0$. Since $\psi$ is $n$-convex, by using Corollary 1 (c), we get (37)-(41). Moreover, the linear function $z$ is convex (concave), therefore considering the positive weights, Remark 1 shows that $0 \leq J_{u}(z) \geq 0$ implying $J_{u}(z)=0$. Finally using the positivity of $J_{u}\left(G_{v}(z, r)\right)$ and (51), (49) is obtained.

(d) Similar to the proof of (c). 


\section{Applications to monotonic functionals}

In [22], Pečarić et al. proposed a more generalized class of $n$-convex functions, that is, $n$-convex functions at a point (see also [1]).

Definition 1 Consider the function $\psi: I \rightarrow \mathbb{R}, d$ is a point in the interior of $I . \psi$ is called $(n+1)$-convex at $d$ if there exists a constant $\mathrm{C}$ so that the function

$$
F(z)=\psi(z)-\frac{C}{n !} z^{n}
$$

is $n$-concave on the interval $I \cap(-\infty, d]$ and $n$-convex on the interval $I \cap[d, \infty)$. The function $\psi$ is called $(n+1)$-concave at $d$ if $-\psi$ is $(n+1)$-convex at $d$.

A property that explains the name of this class is that a function is $n$-convex on an interval if and only if it is $n$-convex at every point of the interval (see [22]). Witkowski et al. in [22] deduced the conditions which are necessary and sufficient on two linear functionals,

$$
\Gamma: C([a, d]) \rightarrow \mathbb{R}
$$

and

$$
\Upsilon: C([d, b]) \rightarrow \mathbb{R}
$$

so that $\Gamma(\psi) \leq \Upsilon(\psi)$ is valid for each $\psi$ which is $n$-convex at $d$. We shall apply this extended class of $n$-convex functions to obtaining monotonic linear functionals of Jensen type. Suppose $\zeta^{u}$ represents the monomials $\zeta^{u}(x)=x^{u}, u \in \mathbb{N}_{0}$. For the remaining part of this section, $\Gamma_{u}\left(\psi_{[a, d]}\right)$ and $\Upsilon_{u}\left(\psi_{[d, b]}\right)$ will represent the linear functionals obtained as the difference of the L.H.S. and R.H.S. of inequality (33), where $u=1, \ldots, 6$, applied to the intervals $[a, d]$ and $[d, b]$, respectively, i.e., for $\mathbf{z} \in[a, d]^{m}$ and $\mathbf{y} \in[d, b]^{m}$. Let

$$
\begin{aligned}
& \Gamma_{u}\left(\psi_{[a, d]}\right):=\Gamma_{u}\left(\mathbf{z}, \mathbf{p}, \lambda ; \psi_{[a, d]}\right)=J_{u}(\psi)-\sum_{w=1}^{n-1} \frac{\psi^{(w)}(a)}{w !} J_{u}\left((z-a)^{w}\right), \\
& \Upsilon_{u}\left(\psi_{[d, b]}\right):=\Upsilon_{u}\left(\mathbf{y}, \mathbf{q}, \tilde{\lambda} ; \psi_{[d, b]}\right)=J_{u}(\psi)-\sum_{w=1}^{n-1} \frac{\psi^{(w)}(d)}{w !} J_{u}\left((y-d)^{w}\right) .
\end{aligned}
$$

In a similar vein, by using the inequality (35), for $u=1, \ldots, 6$, we define linear functionals as

$$
\begin{aligned}
& \hat{\Gamma}_{u}\left(\psi_{[a, d]}\right):=\hat{\Gamma}\left(\mathbf{z}, \mathbf{p}, \lambda ; \psi_{[a, d]}\right)=J_{u}(\psi)-\sum_{w=1}^{n-1} \frac{(-1)^{w} \psi^{(w)}(d)}{w !} J_{u}\left((d-z)^{w}\right), \\
& \hat{\Upsilon}_{u}\left(\psi_{[d, b]}\right):=\hat{\Upsilon}_{u}\left(\mathbf{y}, \mathbf{q}, \tilde{\lambda} ; \psi_{[d, b]}\right)=J_{u}(\psi)-\sum_{w=1}^{n-1} \frac{(-1)^{w} \psi^{(w)}(b)}{w !} J_{u}\left((b-y)^{w}\right) .
\end{aligned}
$$

By constructing new linear functionals $\Gamma_{u}\left(\psi_{[a, d]}\right)$ and $\Upsilon_{u}\left(\psi_{[d, b]}\right)$ using identity (15) for $u=$ $1, \ldots, 6$, on $[a, d]$ and $[d, b]$ we obtain

$$
\Gamma_{u}\left(\psi_{[a, d]}\right)=\frac{1}{(n-1) !} \int_{a}^{d} \psi^{(n)}(\xi) J_{u}\left((z-\xi)_{+}^{n-1}\right) d \xi,
$$




$$
\Upsilon_{u}\left(\psi_{[d, b]}\right)=\frac{1}{(n-1) !} \int_{d}^{b} \psi^{(n)}(\xi) J_{u}\left((y-\xi)_{+}^{n-1}\right) d \xi
$$

By applying identity (16) for $u=1, \ldots, 6$ to the intervals $[a, d]$ and $[d, b]$ we get

$$
\begin{aligned}
& \hat{\Gamma}_{u}\left(\psi_{[a, d]}\right)=-\frac{(-1)^{n-1}}{(n-1) !} \int_{a}^{d} \psi^{(n)}(\xi) J_{u}\left((\xi-z)_{+}^{n-1}\right) d \xi, \\
& \hat{\Upsilon}_{u}\left(\psi_{[d, b]}\right)=-\frac{(-1)^{n-1}}{(n-1) !} \int_{d}^{b} \psi^{(n)}(\xi) J_{u}\left((\xi-y)_{+}^{n-1}\right) d \xi .
\end{aligned}
$$

Immediately, we can state the first theorem of the present section.

Theorem 6 Consider $\mathbf{z} \in[a, d]^{m}$ and $\mathbf{y} \in[d, b]^{m}$ where we have the following.

(i) For $u=1, \ldots, 6$, consider

$$
\begin{aligned}
& J_{u}\left((z-\xi)_{+}^{n-1}\right) \geq 0, \quad \xi \in[a, d], \\
& J_{u}\left((y-\xi)_{+}^{n-1}\right) \geq 0, \quad \xi \in[d, b], \\
& \int_{a}^{d} J_{u}\left((z-\xi)_{+}^{n-1}\right) d \xi=\int_{d}^{b} J_{u}\left((y-\xi)_{+}^{n-1}\right) d \xi,
\end{aligned}
$$

and let $\Gamma_{u}\left(\psi_{[a, d]}\right)$ and $\Upsilon_{u}\left(\psi_{[d, b]}\right)$ be the linear functionals introduced in (54) and (55). If $\psi:[a, b] \rightarrow \mathbb{R}$ is $(n+1)$-convex at the point $d$, then

$$
\Gamma_{u}\left(\psi_{[a, d]}\right) \leq \Upsilon_{u}\left(\psi_{[d, b]}\right) \quad \text { for } u=1, \ldots, 6
$$

(ii) Similarly, for $u=1, \ldots, 6$, suppose

$$
\begin{aligned}
& (-1)^{n-1} J_{u}\left((\xi-z)_{+}^{n-1}\right) \leq 0, \quad \xi \in[a, d], \\
& (-1)^{n-1} J_{u}\left((\xi-y)_{+}^{n-1}\right) \leq 0, \quad \xi \in[d, b], \\
& \int_{a}^{d} J_{u}\left((\xi-z)_{+}^{n-1}\right) d \xi=\int_{d}^{b} J_{u}\left((\xi-y)_{+}^{n-1}\right) d \xi,
\end{aligned}
$$

and let $\hat{\Gamma}_{u}\left(\psi_{[a, d]}\right)$ and $\hat{\Upsilon}_{u}\left(\psi_{[d, b]}\right)$ be the linear functionals which are given by (56) and (57). If $\psi:[a, b] \rightarrow \mathbb{R}$ is $(n+1)$-convex at the point $d$, then

$$
\hat{\Gamma}_{u}\left(\psi_{[a, d]}\right) \leq \hat{\Upsilon}_{u}\left(\psi_{[d, b]}\right) \quad \text { for } u=1, \ldots, 6
$$

Proof (i) Fix $u=1, \ldots, 6$. Using Definition 1 , construct the function $F=\psi-\frac{C}{n !} \zeta^{n}$ such that $F$ is $n$-concave on $[a, d]$ and is $n$-convex on $[d, b]$. Now by applying Corollary 1 to $F$ in the interval $[a, d]$, we get

$$
0 \geq \Gamma_{u}(\Psi)=\Gamma_{u}\left(\psi_{[a, d]}\right)-\frac{\mathrm{C}}{(n) !} \Gamma_{u}\left(\zeta_{[a, d]}^{n}\right) .
$$

Similarly, applying Corollary 1 to $F$ in $[d, b]$, we have

$$
0 \leq \Upsilon_{u}(\Psi)=\Upsilon_{u}\left(\psi_{[d, b]}\right)-\frac{\mathrm{C}}{(n) !} \Upsilon_{u}\left(\zeta_{[d, a]}^{n}\right)
$$


Moreover, utilizing the monomials $\zeta^{n}(\cdot)$ in (58) and (59) we get

$$
\begin{aligned}
& \Gamma_{u}\left(\zeta_{[a, d]}^{n}\right)=n \int_{a}^{d} J_{u}\left((z-\xi)_{+}^{n-1}\right) d \xi \\
& \Upsilon_{u}\left(\zeta_{[d, b]}^{n}\right)=n \int_{d}^{b} J_{u}\left((y-\xi)_{+}^{n-1}\right) d \xi
\end{aligned}
$$

Therefore assumption (64) is equivalent to

$$
\Gamma_{u}\left(\zeta_{[a, d]}^{n}\right)=\Upsilon_{u}\left(\zeta_{[d, b]}^{n}\right)
$$

So from (70) and (71), one can get

$$
\Gamma_{u}\left(\psi_{[a, d]}\right) \leq \frac{\mathrm{C}}{(n) !} \Gamma_{u}\left(\zeta_{[a, d]}^{n}\right)=\frac{\mathrm{C}}{(n) !} \Upsilon_{u}\left(\zeta_{[d, b]}^{n}\right) \leq \Upsilon_{u}\left(\psi_{[d, b]}\right)
$$

So (65) is obtained for $u=1, \ldots, 6$.

(ii) A method similar to the above can be employed by using the identities (60) and (61). Hence by deducing supposition (68), we have (69) for $u=1, \ldots, 6$.

We conclude with the following remarks.

Remark 2 Note that inequality (65) and (69) are also valid on replacing assumptions (64) and (68) with the weaker assumptions that

$$
\begin{aligned}
& \mathrm{C}\left(\Upsilon_{u}\left(\zeta_{[d, b]}^{n}\right)-\Gamma_{u}\left(\zeta_{[a, d]}^{n}\right)\right) \geq 0 \quad \text { and } \\
& \mathrm{C}\left(\hat{\Upsilon}_{u}\left(\zeta_{[d, b]}^{n}\right)-\hat{\Gamma}_{u}\left(\zeta_{[a, d]}^{n}\right)\right) \geq 0
\end{aligned}
$$

for $u=1, \ldots, 6$, respectively.

Remark 3 Similar results can also be formulated by employing Theorem 6 for the inequalities (37) to (41) and (43) to (47) in Corollary 1.

\section{New entropic bounds}

In fields like probability theory, mathematical statistics and information theory, measures of dissimilarity between probability distributions play a pivotal role. Various divergence measures have been introduced for this purpose. For instance, we have the $f$ divergence, some particular cases of which are the Kullback-Leibler divergence and the Jensen-Shannon divergence. Entropies are used to quantify the uncertainty, diversity and the randomness of a system. The idea is frequently used in several scientific disciplines. Some new estimations for the Shannon and Zipf-Mandelbrot entropies can be found in [14].

In the current section we will work in discrete space, i.e., with discrete probability distributions.

We first introduce some important definitions and results used for the rest of this section. Csiszár in [5] introduced the following notion of a divergence functional. 
Definition 2 Let $\psi:(0, \infty) \rightarrow(0, \infty)$ be a convex function and let $\mathbf{p}:=\left(p_{1}, \ldots, p_{m}\right)$ and $\mathbf{q}:=\left(q_{1}, \ldots, q_{m}\right)$ be positive probability distributions. The $\psi$-divergence functional is

$$
I_{\psi}(\mathbf{p}, \mathbf{q}):=\sum_{i=1}^{n} q_{i} \psi\left(\frac{p_{i}}{q_{i}}\right)
$$

It is possible to use non-negative probability distributions in the $\psi$-divergence functional, by defining

$$
\psi(0):=\lim _{t \rightarrow 0+} \psi(t) ; \quad 0 \psi\left(\frac{0}{0}\right):=0 ; \quad 0 \psi\left(\frac{a}{0}\right):=\lim _{t \rightarrow 0+} t \psi\left(\frac{a}{t}\right), \quad a>0 .
$$

Based on the previous definition, we introduce a new functional.

Definition 3 Suppose $\psi: I \rightarrow \mathbb{R}$ with $I$ being an interval in $\mathbb{R}$. Suppose $\mathbf{p}:=\left(p_{1}, \ldots, p_{m}\right) \in$ $\mathbb{R}^{m}$ and $\mathbf{q}:=\left(q_{1}, \ldots, q_{m}\right) \in(0, \infty)^{m}$ such that

$$
\frac{p_{i}}{q_{i}} \in I, \quad i=1, \ldots, m
$$

Then let

$$
\widetilde{I}_{\psi}(\mathbf{p}, \mathbf{q})=\sum_{i=1}^{m} q_{i} \psi\left(\frac{p_{i}}{q_{i}}\right)
$$

Remark 4 As a consequences of Corollary 1 for the case $u=1$, we consider the explicit form of our generalized Jensen inequalities for $n$-convex functions, from (33) and (35):

$$
\begin{aligned}
& \sum_{i=1}^{m} p_{i} \psi\left(z_{i}\right)-\sum_{i=1}^{m}\left(\sum_{j=0}^{k-1} \lambda_{j+1} p_{i+j}\right) \psi\left(\frac{\sum_{j=0}^{k-1} \lambda_{j+1} p_{i+j} z_{i+j}}{\sum_{j=0}^{k-1} \lambda_{j+1} p_{i+j}}\right) \\
& \geq \sum_{w=1}^{n-1} \frac{\psi^{(w)}(a)}{w !}\left(\sum_{i=1}^{m} p_{i}\left(z_{i}-a\right)^{w}-\sum_{i=1}^{m}\left(\sum_{j=0}^{k-1} \lambda_{j+1} p_{i+j}\right)\left(\frac{\sum_{j=0}^{k-1} \lambda_{j+1} p_{i+j} z_{i+j}}{\sum_{j=0}^{k-1} \lambda_{j+1} p_{i+j}}-a\right)^{w}\right), \quad(76) \\
& \sum_{i=1}^{m} p_{i} \psi\left(z_{i}\right)-\sum_{i=1}^{m}\left(\sum_{j=0}^{k-1} \lambda_{j+1} p_{i+j}\right) \psi\left(\frac{\sum_{j=0}^{k-1} \lambda_{j+1} p_{i+j} z_{i+j}}{\sum_{j=0}^{k-1} \lambda_{j+1} p_{i+j}}\right) \\
& \geq \sum_{w=1}^{n-1} \frac{(-1)^{w} \psi^{(w)}(b)}{w !}\left(\sum_{i=1}^{m} p_{i}\left(b-z_{i}\right)^{w}-\sum_{i=1}^{m}\left(\sum_{j=0}^{k-1} \lambda_{j+1} p_{i+j}\right)\left(b-\frac{\sum_{j=0}^{k-1} \lambda_{j+1} p_{i+j} z_{i+j}}{\sum_{j=0}^{k-1} \lambda_{j+1} p_{i+j}}\right)^{w}\right) .
\end{aligned}
$$

For the rest of this section, we will use the following assumptions:

$\left(\mathrm{A}_{3}\right)$ Suppose for $m, k \in \mathbb{N}(2 \leq k \leq m), \lambda_{1}, \ldots, \lambda_{k}$ are positive probability distributions.

$\left(\mathrm{A}_{4}\right)$ Let $\mathbf{q}:=\left(q_{1}, \ldots, q_{m}\right) \in(0, \infty)^{m}$.

Theorem 7 Considering $\left(\mathrm{A}_{3}\right)$ and $\left(\mathrm{A}_{4}\right)$, let $\mathbf{p}:=\left(p_{1}, \ldots, p_{m}\right) \in \mathbb{R}^{m}$ such that

$$
\frac{p_{i}}{q_{i}} \in[a, b], \quad i=1, \ldots, m
$$


Let $\psi:[a, b] \rightarrow \mathbb{R}$ where $\psi^{(n-1)}$ is absolutely continuous and $\psi$ is $n$-convex, then the following inequalities hold:

(a)

$$
\begin{aligned}
\widetilde{I}_{\psi}(\mathbf{p}, \mathbf{q}) \geq & \sum_{i=1}^{m}\left(\sum_{j=0}^{k-1} \lambda_{j+1} q_{i+j}\right) \psi\left(\frac{\sum_{j=0}^{k-1} \lambda_{j+1} p_{i+j}}{\sum_{j=0}^{k-1} \lambda_{j+1} q_{i+j}}\right)+\sum_{w=1}^{n-1} \frac{\psi^{(w)}(a)}{w !} \\
& \cdot\left(\sum_{i=1}^{m} q_{i}\left(\frac{p_{i}}{q_{i}}-a\right)^{w}-\sum_{i=1}^{m}\left(\sum_{j=0}^{k-1} \lambda_{i+1} q_{i+j}\right)\left(\frac{\sum_{i=0}^{k-1} \lambda_{i+1} p_{i+j}}{\sum_{j=0}^{k-1} \lambda_{j+1} q_{i+j}}-a\right)^{w}\right),
\end{aligned}
$$

(b)

$$
\begin{aligned}
\widetilde{I}_{\psi}(\mathbf{p}, \mathbf{q}) \geq & \sum_{i=1}^{m}\left(\sum_{j=0}^{k-1} \lambda_{j+1} q_{i+j}\right) \psi\left(\frac{\sum_{j=0}^{k-1} \lambda_{j+1} p_{i+j}}{\sum_{j=0}^{k-1} \lambda_{j+1} q_{i+j}}\right)+\sum_{w=1}^{n-1} \frac{(-1)^{w} \psi^{(w)}(b)}{w !} \\
& \cdot\left(\sum_{i=1}^{m} q_{i}\left(b-\frac{p_{i}}{q_{i}}\right)^{w}-\sum_{i=1}^{m}\left(\sum_{j=0}^{k-1} \lambda_{j+1} q_{i+j}\right)\left(b-\frac{\sum_{j=0}^{k-1} \lambda_{j+1} p_{i+j}}{\sum_{j=0}^{k-1} \lambda_{j+1} q_{i+j}}\right)^{w}\right) .
\end{aligned}
$$

Proof Replacing $p_{i}$ with $q_{i}$ and $z_{i}$ with $\frac{p_{i}}{q_{i}}$ for $(i=1, \ldots, m)$ in (76) and (77), we get (78) and (79), respectively.

We now present two significant applications of the previous results.

Shannon's entropy, also known as a measure of uncertainty, plays a pivotal role in information theory. It is also frequently applied in fields like population genetics, molecular ecology and dynamical systems (see [18]). Some recent bounds for Shannon entropy can be found in $[9,15]$. For a positive $m$-tuple $\mathbf{p}=\left(p_{1}, \ldots, p_{m}\right)$ such that $\sum_{i=1}^{m} p_{i}=1$, the Shannon entropy is defined by

$$
S(\mathbf{p})=-\sum_{i=1}^{m} p_{i} \ln p_{i} .
$$

\section{Corollary 2 Suppose $\left(\mathrm{A}_{3}\right)$ holds.}

(a) Considering $\left(\mathrm{A}_{4}\right)$, if $n$ is even, we get

$$
\begin{aligned}
\sum_{i=1}^{m} q_{i} \ln q_{i} \geq & \sum_{i=1}^{m}\left(\sum_{j=0}^{k-1} \lambda_{j+1} q_{i+j}\right) \ln \left(\sum_{j=0}^{k-1} \lambda_{j+1} q_{i+j}\right)+\sum_{w=1}^{n-1} \frac{(-1)^{w}}{w \cdot(a)^{w}} \\
& \cdot\left(\sum_{i=1}^{m} q_{i}\left(\frac{1}{q_{i}}-a\right)^{w}-\sum_{i=1}^{m}\left(\sum_{j=0}^{k-1} \lambda_{j+1} q_{i+j}\right)\left(\frac{1}{\sum_{j=0}^{k-1} \lambda_{j+1} q_{i+j}}-a\right)^{w}\right) .
\end{aligned}
$$

(b) If $\mathbf{q}:=\left(q_{1}, \ldots, q_{m}\right)$ is a positive probability distribution, while $n$ is even, we obtain the bounds for the Shannon entropy of $\mathbf{q}$,

$$
\begin{aligned}
S(\mathbf{q}) \leq & -\sum_{i=1}^{m}\left(\sum_{j=0}^{k-1} \lambda_{j+1} q_{i+j}\right) \ln \left(\sum_{j=0}^{k-1} \lambda_{j+1} q_{i+j}\right)-\sum_{w=1}^{n-1} \frac{(-1)^{w}}{w \cdot(a)^{w}} \\
& \cdot\left(\sum_{i=1}^{m} q_{i}\left(\frac{1}{q_{i}}-a\right)^{w}-\sum_{i=1}^{m}\left(\sum_{j=0}^{k-1} \lambda_{j+1} q_{i+j}\right)\left(\frac{1}{\sum_{j=0}^{k-1} \lambda_{j+1} q_{i+j}}-a\right)^{w}\right) .
\end{aligned}
$$

For $n$ odd, (81) and (82) are satisfied in the reverse directions. 
Proof (a) Substituting $\psi(t):=-\ln t$, and $\mathbf{p}:=(1,1, \ldots, 1)$ in Theorem $7(\mathrm{a})$, we obtain the desired results.

(b) This part is a special case of (a).

The Kullback-Leibler measure, also known as the relative entropy, is a famous nonparametric measure. It is also one of the most famous distance functions used in signal processing, information theory and mathematical statistics. Some recent bounds for the relative entropy can be found in [9, 15]. The Kullback-Leibler distance [17] between the positive probability distributions $\mathbf{p}=\left(p_{1}, \ldots, p_{m}\right)$ and $\mathbf{q}=\left(q_{1}, \ldots, q_{m}\right)$ is given as

$$
D(\mathbf{q} \| \mathbf{p})=\sum_{i=1}^{m} q_{i} \ln \left(\frac{q_{i}}{p_{i}}\right) .
$$

Corollary 3 Suppose $\left(\mathrm{A}_{3}\right)$ holds.

(a) If $\mathbf{q}:=\left(q_{1}, \ldots, q_{m}\right), \mathbf{p}:=\left(p_{1}, \ldots, p_{m}\right) \in(0, \infty)^{m}$. Then, for even $n$, we get

$$
\begin{aligned}
\sum_{i=1}^{m} q_{i} \ln \left(\frac{q_{i}}{p_{i}}\right) \geq & \sum_{i=1}^{m}\left(\sum_{j=0}^{k-1} \lambda_{j+1} q_{i+j}\right) \ln \left(\frac{\sum_{j=0}^{k-1} \lambda_{j+1} q_{i+j}}{\sum_{j=0}^{k-1} \lambda_{j+1} p_{i+j}}\right)+\sum_{w=1}^{n-1} \frac{(-1)^{w}}{w \cdot(a)^{w}} \\
& \cdot\left(\sum_{i=1}^{m} q_{i}\left(\frac{p_{i}}{q_{i}}-a\right)^{w}-\sum_{i=1}^{m}\left(\sum_{j=0}^{k-1} \lambda_{j+1} q_{i+j}\right)\left(\frac{\sum_{j=0}^{k-1} \lambda_{j+1} p_{i+j}}{\sum_{j=0}^{k-1} \lambda_{j+1} q_{i+j}}-a\right)^{w}\right) .
\end{aligned}
$$

(b) If $\mathbf{q}:=\left(q_{1}, \ldots, q_{m}\right), \mathbf{p}:=\left(p_{1}, \ldots, p_{m}\right)$ are positive probability distributions with $n$ even, we obtain

$$
\begin{aligned}
D(\mathbf{q} \| \mathbf{p}) \geq & \sum_{i=1}^{m}\left(\sum_{j=0}^{k-1} \lambda_{j+1} q_{i+j}\right) \ln \left(\frac{\sum_{j=0}^{k-1} \lambda_{j+1} q_{i+j}}{\sum_{j=0}^{k-1} \lambda_{j+1} p_{i+j}}\right)+\sum_{w=1}^{n-1} \frac{(-1)^{w}}{w \cdot(a)^{w}} \\
& \cdot\left(\sum_{i=1}^{m} q_{i}\left(\frac{p_{i}}{q_{i}}-a\right)^{w}-\sum_{i=1}^{m}\left(\sum_{j=0}^{k-1} \lambda_{j+1} q_{i+j}\right)\left(\frac{\sum_{j=0}^{k-1} \lambda_{j+1} p_{i+j}}{\sum_{j=0}^{k-1} \lambda_{j+1} q_{i+j}}-a\right)^{w}\right) .
\end{aligned}
$$

In the case $n$ is odd, (84) and (85) are satisfied in the reverse directions.

Proof (a) Setting $\psi(t):=-\ln t$ in Theorem $7($ a) gives the required results.

(b) This part is a special case of (a).

Philologist George Kingsley Zipf (1902-1950) studied statistical occurrences of words in different languages. It was one of the first academic studies of word frequency and was originally prescribed only for linguistics. It was only later that many other disciplines took credit of it: the Pareto law in economy reveals another aspect of it and the "Zipfian distribution" is present in other fields as well: information science, bibliometrics, social sciences etc.

Benoit Mandelbrot (1924-2010), in 1966, generalized the Zipf law and improved for counting the low-rank words. It has various applications in art, in ecological field studies and is also used in information sciences [6, p. 294]. Some of the recent studies regarding the Zipf-Mandelbrot law can be found in the listed references (see $[9,10,15,16])$. 
Hence if we rank $(r)$ word in accordance with the frequency of the word occurrence $(\psi)$, the product is a constant $(C)$ :

$$
C=r \psi
$$

For $m \in\{1,2, \ldots\}, t \geq 0$ and $s>0$ the Zipf-Mandelbrot law is given as

$$
\psi(i ; m, t, s)=\frac{1}{\left((i+t)^{s} H_{t, s}^{m}\right)}, \quad i=(1,2, \ldots, m)
$$

where

$$
H_{t, s}^{m}=\sum_{k=1}^{m} \frac{1}{(k+t)^{s}}
$$

In the formula, $i$ represents the rank of the data, $t$ and $s$ are parameters of the distribution. Suppose $m \in\{1,2, \ldots\}, t \geq 0, s>0$, then the Zipf-Mandelbrot entropy is given as

$$
Z(H, t, s)=\frac{s}{H_{t, s}^{m}} \sum_{i=1}^{m} \frac{\ln (i+t)}{(i+t)^{s}}+\ln \left(H_{t, s}^{m}\right) .
$$

\section{Consider}

$$
q_{i}=\psi(i ; m, t, s)=\frac{1}{\left((i+t)^{s} H_{t, s}^{m}\right)} .
$$

The following results give bounds for the Zipf-Mandelbrot entropy.

Theorem 8 Suppose $\left(\mathrm{A}_{3}\right)$ holds and $\mathbf{q}$ is the same as given in (88) by the Zipf-Mandelbrot law with parameters $m \in\{1,2, \ldots\}, t \geq 0, s>0$. For $n$ even, we have

$$
\begin{aligned}
S(\mathbf{q})= & Z(H, t, s) \\
\leq & -\sum_{i=1}^{m}\left(\sum_{j=0}^{k-1} \frac{\lambda_{j+1}}{\left((i+j+t)^{s} H_{t, s}^{m}\right)}\right) \ln \left(\frac{1}{H_{t, s}^{m}} \sum_{j=0}^{k-1} \frac{\lambda_{j+1}}{\left((i+j+t)^{s}\right)}\right) \\
& -\sum_{w=1}^{n-1} \frac{(-1)^{w}}{w \cdot(a)^{w}}\left(\sum_{i=1}^{m} \frac{1}{\left((i+t)^{s} H_{t, s}^{m}\right)}\left(\left((i+t)^{s} H_{t, s}^{m}\right)-a\right)^{w}\right) \\
& +\sum_{w=1}^{n-1} \frac{(-1)^{w}}{w \cdot(a)^{w}}\left(\sum_{i=1}^{m}\left(\sum_{j=0}^{k-1} \frac{\lambda_{j+1}}{\left((i+j+t)^{s} H_{t, s}^{m}\right)}\right)\left(\frac{1}{\sum_{j=0}^{k-1} \frac{\lambda_{j+1}}{\left((i+j+t)^{s} H_{t, s}^{m}\right)}}-a\right)^{w}\right) .
\end{aligned}
$$

Equation (89) holds in the opposite direction for $n$ odd.

Proof Substituting this $q_{i}=\frac{1}{\left((i+t)^{s} H_{t, s}^{m}\right)}$ in Corollary 2(b) gives the required result. Also note that $\sum_{i=1}^{m} q_{i}=1$. Moreover, using the above $q_{i}$ in the Shannon entropy (80), we get the 
Mandelbrot entropy (87),

$$
\begin{aligned}
S(q) & =-q_{i} \ln q_{i} \\
& =-\sum_{i=1}^{m} \frac{1}{\left((i+t)^{s} H_{t, s}^{m}\right)} \ln \frac{1}{\left((i+t)^{s} H_{t, s}^{m}\right)} \\
& =\frac{-1}{H_{t, s}^{m}} \sum_{i=1}^{m} \frac{1}{(i+t)^{s}} \ln \frac{1}{(i+t)^{s} H_{t, s}^{m}} \\
& =\frac{-1}{H_{t, s}^{m}} \sum_{i=1}^{m} \frac{1}{(i+t)^{s}}\left(\ln (1)-s \ln (u+t)-\ln \left(H_{t, s}^{m}\right)\right) \\
& =\frac{1}{H_{t, s}^{m}} \sum_{i=1}^{m} \frac{1}{(i+t)^{s}}\left(s \ln (i+t)+\ln \left(H_{t, s}^{m}\right)\right) \\
& =\frac{s}{H_{t, s}^{m}} \sum_{i=1}^{m} \frac{\ln (i+t)}{(i+t)^{s}}+\ln \left(H_{t, s}^{m}\right) .
\end{aligned}
$$

Corollary 4 Suppose $\left(\mathrm{A}_{3}\right)$ holds and for $t_{1}, t_{2} \in[0, \infty), s_{1}, s_{2}>0$, let $H_{t_{1}, s_{1}}^{m}=\sum_{k=1}^{m} \frac{1}{\left(k+t_{1}\right)^{s_{1}}}$ and $H_{t_{2}, s_{2}}^{m}=\sum_{k=1}^{m} \frac{1}{\left(k+t_{2}\right)^{s_{2}}}$. Now using $q_{i}=\frac{1}{\left(i+t_{1}\right)^{s_{1}} H_{t_{1}, s_{1}}^{m}}$ and $p_{i}=\frac{1}{\left(i+t_{2}\right)^{s_{2}} H_{t_{2}, s_{2}}^{m}}$ in Corollary 3(b), with $n$ even, we have

$$
\begin{aligned}
D(\mathbf{q} \| \mathbf{p})= & \sum_{i=1}^{m} \frac{1}{\left(i+t_{1}\right)^{s_{1}} H_{t_{1}, s_{1}}^{m}} \ln \left(\frac{\left(i+t_{2}\right)^{s_{2}} H_{t_{2}, s_{2}}^{m}}{\left(i+t_{1}\right)^{s_{1}} H_{t_{1}, s_{1}}^{m}}\right) \\
\geq & \sum_{i=1}^{m}\left(\sum_{j=0}^{k-1} \frac{\lambda_{j+1}}{\left(i+j+t_{1}\right)^{s_{1}} H_{t_{1}, s_{1}}^{m}}\right) \ln \left(\frac{\sum_{j=0}^{k-1} \lambda_{j+1} \frac{1}{\left(i+j+t_{1}\right)^{s_{1}} H_{t_{1}, s_{1}}^{m}}}{\sum_{j=0}^{k-1} \lambda_{j+1} \frac{1}{\left(i+j+t_{2}\right)^{s_{2}} H_{t_{2}, s_{2}}^{m}}}\right) \\
& +\sum_{w=1}^{n-1} \frac{(-1)^{w}}{w \cdot(a)^{w}}\left(\sum_{i=1}^{m} \frac{1}{\left(i+t_{1}\right)^{s_{1}} H_{t_{1}, s_{1}}^{m}}\left(\frac{\left(i+t_{1}\right)^{s_{1}} H_{t_{1}, s_{1}}^{m}}{\left(i+t_{2}\right)^{s_{2}} H_{t_{2}, s_{2}}^{m}}-a\right)^{w}\right) \\
& -\sum_{w=1}^{n-1} \frac{(-1)^{w}}{w \cdot(a)^{w}} \\
& \cdot\left(\sum_{i=1}^{m}\left(\sum_{j=0}^{k-1} \frac{\lambda_{j+1}}{\left(i+j+t_{1}\right)^{s_{1}} H_{t_{1}, s_{1}}^{m}}\right)\left(\frac{\sum_{j=0}^{k-1} \lambda_{j+1} \frac{1}{\left(i+j+t_{2}\right)^{s_{2}} H_{t_{2}, s_{2}}^{m}}}{\sum_{j=0}^{k-1} \lambda_{j+1} \frac{1}{\left(i+j+t_{1}\right)^{s_{1}} H_{t_{1}, s_{1}}^{m}}}-a\right)^{w}\right) .
\end{aligned}
$$

If $n$ is odd, (91) holds in the reverse direction.

\section{Concluding remarks}

New upper bounds for Grïss and Ostrowski type inequalities can be formulated (see [2]) as a direct application of the results obtained by Dragomir et al. in [4] from new generalized inequalities for $n$-convex functions obtained in the first section. We can also construct several functionals from the inequalities introduced in Theorem 1 and give mean value theorems of Lagrange and Cauchy type for $n$-convex functions. Moreover, considering the $n$-exponentially method introduced by Pečarić et al. in [11] and [21] (see also [3]), we can establish a novel collection of non-trivial examples of functions which are $n$-exponentially and exponentially convex. Finally we can also construct monotonic Cauchy means. 


\section{Acknowledgements}

The research of first and second author is supported by Higher Education Commission (H.E.C.) under National Research project (NRPU 7906). The research of the fourth author is supported by the Ministry of Education and Science of the Russian Federation (Agreement number No. 02.a03.21.0008).

\section{Funding}

The research is completely supported by Higher Education Commission (H.E.C.) of Pakistan under NRPU project 7906.

\section{Competing interests}

The authors declare that they have no competing interests.

\section{Authors' contributions}

All authors jointly worked on the results and they read and approved the final manuscript.

\section{Author details}

'Department of Mathematics, COMSATS University Islamabad, Lahore Campus, Pakistan. ${ }^{2}$ Catholic University of Croatia, Zagreb, Croatia. ${ }^{3}$ RUDN University, Moscow, Russia.

\section{Publisher's Note}

Springer Nature remains neutral with regard to jurisdictional claims in published maps and institutional affiliations.

Received: 30 May 2019 Accepted: 27 August 2019 Published online: 06 September 2019

\section{References}

1. Baloch, I.A., Pečarić, J., Praljak, M.: Generalization of Levinson's inequality. J. Math. Inequal. 9, 571-586 (2015)

2. Butt, S.I., Pečarić, J.: Popoviciu's Inequality for N-Convex Functions. Lap Lambert Academic Publishing, Saarbrücken (2016). ISBN:978-3-659-81905-6

3. Butt, S.I., Pečarić, J., Vukelić, A.: Generalization of Popoviciu type inequalities via Fink's identity. Mediterr. J. Math. 13(4), 1495-1511 (2016)

4. Cerone, P., Dragomir, S.S.: Some new Ostrowski-type bounds for the Čebyšev functional and applications. J. Math. Inequal. 8(1), 159-170 (2014)

5. Csiszár, I.: Information-type measures of diference of probability distributions and indirect observations. Studia Sci. Math. Hung. 2, 299-318(1967)

6. Egghe, L., Rousseau, R.: Introduction to Informetrics. Quantitative Methods in Library, Documentation and Information Science. Elsevier, New York (1990)

7. Farid, G., Katugampola, U.N.: Ostrowski type fractional integral inequalities for S-Godunova-Levin functions via Katugampola fractional integrals. Open J. Math. Sci. 1(1), 97-110 (2017)

8. Horváth, L., Khan, K.A., Pečarić, J.: Cyclic refinements of the discrete and integral form of Jensen's inequality with applications. Analysis (Munich) 36(4), 253-263 (2016)

9. Horváth, L., Pečarić, Đ., Pečarić, J.: Estimations of $f$ - and Rényi divergences by using a cyclic refinement of the Jensen's inequality. Bull. Malays. Math. Sci. Soc. (2017). https://doi.org/10.1007/s40840-017-0526-4

10. Jakšetic, J., Pečarić, Đ., Pečarić, J.: Some properties of Zipf-Mandelbrot law and Hurwitz $\zeta$-function. Math. Inequal. Appl. 21(2), 575-584 (2018)

11. Jakšetic, J., Pečarić, J.: Exponential convexity method. J. Convex Anal. 20(1), 181-197 (2013)

12. Kermausuor, S.: Simpson's type inequalities for strongly $(s, m)$-convex functions in second sense and applications. Open J. Math. Sci. 3(1), 74-83 (2019)

13. Khan, J., Khan, M.A., Pečarić, J.: On Jensen's type inequalities via generalized majorization inequalities. Filomat 32(16), 5719-5733 (2018)

14. Khan, M.A., Al-sahwi, Z.M., Chu, Y.M.: New estimations for Shannon and Zipf-Mandelbrot entropies. Entropy 20, 608 (2018)

15. Khan, M.A., Pečarić, Đ., Pečarić, J.: Bounds for Shannon and Zipf-Mandelbrot entropies. Math. Methods Appl. Sci. 40(18), 7316-7322 (2017)

16. Khan, M.A., Pečarić, Đ., Pečarić, J.: On Zipf-Mandelbrot entropy. Comput. Appl. Math. 346, 192-204 (2019)

17. Kullback, S., Leibler, R.A.: On information and sufficiency. Ann. Math. Stat. 22, 79-86 (1951)

18. Lesne, A.: Shannon entropy: a rigorous notion at the crossroads between probability, information theory, dynamical systems and statistical physics. Math. Struct. Comput. Sci. 24(3), e240311 (2014) https://doi.org/10.1017/S0960129512000783

19. Mehmood, N., Agarwal, R.P., Butt, S.I., Pečarić, J.: New generalizations of Popoviciu-type inequalities via new Green's functions and Montgomery identity. J. Inequal. Appl. 2017, 108 (2017)

20. Moradi, H.R., Omidvar, M.E., Khan, M.A., Nickodem, K.: Around Jensen's inequality for strongly convex functions. Aequ. Math. 92, 25-37 (2018)

21. Pečarić, J., Perić, J.: Improvement of the Giaccardi and the Petrović inequality and related Stolarsky type means. An. Univ. Craiova, Ser. Mat. Inform. 39(1), 65-75 (2012)

22. Pečarić, J., Praljak, M., Witkowski, A.: Linear operator inequality for $n$-convex functions at a point. Math. Inequal. Appl. 18, 1201-1217 (2015)

23. Pečarić, J., Proschan, F., Tong, Y.L.: Convex Functions, Partial Orderings and Statistical Applications. Academic Press, New York (1992)

24. Saleem, M.S., Pečarić, J., Munir, M., Ali, A., Tubssam, M.S.I.: The weighted square integral inequalities for smooth and weak subsolution of fourth order Laplace equation. Open J. Math. Sci. 2(1), 228-239 (2018) 